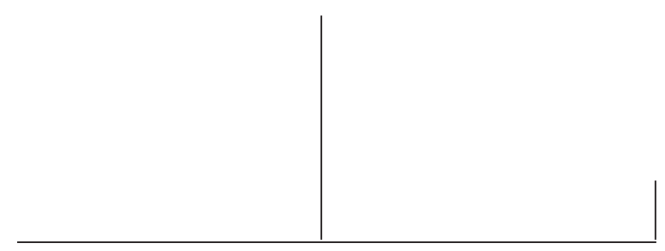

Rev. Latinoam. Psicopat. Fund., V, 4, 45-62

\title{
L'elaboration subjective des liens en question. Représentation des liens de parenté au génogramme
}

Marie-José Grihom, A. Laflaquiere e

A. Ducousso-Lacaze

\begin{abstract}
L'auteur propose d'aborder l'éventuelle "nouveauté" des liens familiaux pour la psyché à partir de ce que des enfants-sujets peuvent en élaborer subjectivement. Il développe l'idée selon laquelle la qualité de l'élaboration subjective des liens de parenté au génogramme est significative de la manière dont le sujet subjective sa position instituée et sa place inconsciente dans le lien avec l'autre. Il ressort au travers d'une situation clinique que les modes de tension entre connaissance et représentation des liens sont significatifs $d u$ travail de l'Oedipe chez le sujet.
\end{abstract}

Mots clés: Élaboration subjective, lien, connaissance, représentation, génogramme 


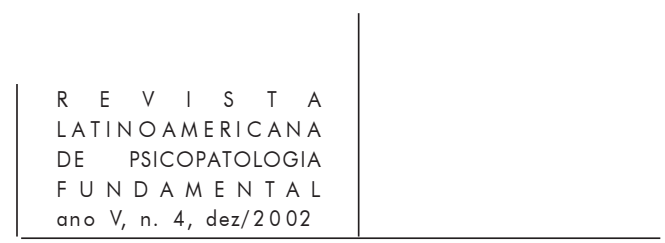

\section{Introduction}

Cette communication prend appui sur un travail de recherche dont l'objet central est l'élaboration subjective des liens de parenté dans l'enfance.

En bref, l'élaboration par le sujet de ses liens et de sa place en leur sein serait en question dans la mesure où le/les liens familiaux feraient l'objet de remaniements, de changements et ce tant du point de vue social que psychologique. Cette inférence entre une forme d'innovation structurelle ou fonctionnelle des liens familiaux (selon que l'on interroge par exemple la pérennité de la fonction paternelle dans les familles contemporaines ou l'effet des rôles et des statuts sociaux sur la construction de l'être parent) et un risque ou un dommage pour la construction subjective, traverse si nettement nos discours contemporains qu'elle mérite que l'on s'y arrête. L'élaboration subjective des liens estelle "menacée" par la nouveauté laissant le sujet dans un trop de liens inélaborable ou dans un défaut de repères symboliques? La nouveauté du lien, qui irait de pair avec l'évolution complexe des représentations culturelles de la double différence, génère-t-elle de l'incertain voire de l'impensable chez le sujet avec en fond un rapport à l'interdit et à la castration problématiques?

Nous avons choisi de nous poser à ce carrefour si complexe entre le champ social (et les formes du lien social) et le champ de l'intersubjectivité munie de quelques outils: ceux d'une psychologie clinique de la construction du sujet et ceux d'une conception freudienne de l'élaboration mentale et psychique. Aucune espèce de causalité directe 


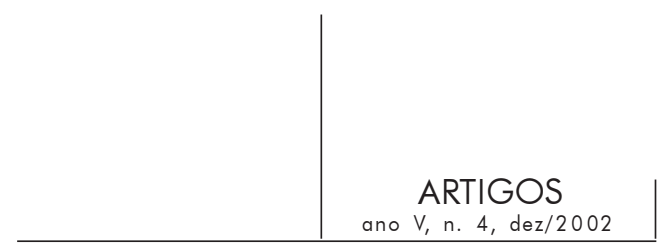

entre le réalisant du lien qu'est le parent du sujet et la construction subjective de celui-ci n'étant possible, nous sommes partie de l'idée selon laquelle la caractéristique du lien familial tient à son hétérogénéité même (Guyotat, 1980, 1993) et que celle-ci a pour conséquence de susciter ou d'imposer au sujet une somme de travail psychique du fait même de la liaison de la psyché à la pulsion (Freud, 1905/1990), à la culture et à l'autre (Freud, 1912-1913/1980; Legendre, 1985, 1990; Kaës, 1993a, 1993b).

A la suite des travaux sur la construction de la parenté par le sujet dans le cadre du cycle de recomposition familiale (Hurstel, 1994), de ceux de M.L. Pradelles-Monod $(1998,1999)$ sur la construction du lien de filiation entre trois générations, et de nos recherches communes (Ducousso-Lacaze e Grihom, 2001; Laflaquiere, 2001), nous adoptons l'option qui consiste à dire que toute forme de lien entre le sujet et l'autre dans le champ de la parenté - et ce quelle que soit pour le sujet la part prise par les paroles qui instituent le lien, l'imaginarisent ou l'ancestralisent (Pradelles-Monod, 1994, 2000) - est source de travail psychique et que le récit ou la représentation qu'en produit le sujet est le témoin de ce travail.

Dans le cadre qui est le nôtre ce témoin empirique de l'élaboration subjective du lien ou de son impossibilité à être élaboré, réside dans les modalités de mise en représentation par le sujet de sa généalogie lors de la réalisation de son génogramme. Mixte de signifiants iconiques et verbaux inscrits ou énoncés, le génogramme est une production sémiotique en même temps qu'un “texte" secondarisé, comme l'est le récit du rêve. Dans ce "texte" le sujet se situe dans ses liens dans l'instant même où il s'y représente à partir des logiques intrasubjectives et intersubjectives de désir qui sont les siennes. Aussi nous semble-t-il significatif de la manière dont le "sujet du lien" peut ou non disposer d'un espace pour penser le possible de sa condition d'être humain mortel et sexué. Cet espace du penser (de l'élaboration subjective) étant limité sur un bord par le champ de l'interdit et de la réalité sociale, pénétré sur l'autre bord par le champ du désirable et de la subversion du désir nous semble témoigner du degré d'élaboration de la problématique oedipienne. Selon ce que le sujet dit et figure analogiquement de sa place dans les liens au génogramme nous pouvons ainsi tout à la fois inférer les représentations conscientes et inconscientes à l'œuvre et saisir les processus concourant à l'élaboration subjective (les modes de tension entre processus primaire et secondaire, les parts respectives du fantasme et de l'interdit).

Deux questions centrales de recherche se dégagent en conséquence. 1) Comment le sujet représente-t-il sa position dans la configuration relationnelle qu'est son groupe familial notamment selon la composition de son groupe de vie et la qualité de son environnement familial au sens winnicottien du terme? 


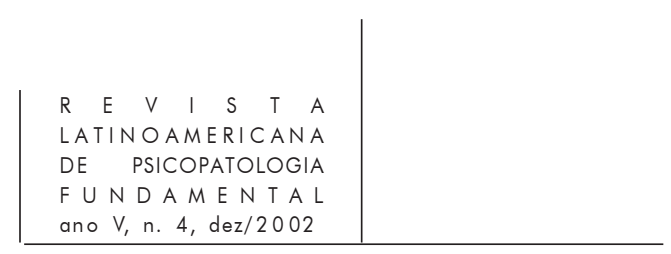

Autrement dit l'élaboration subjective est-elle en relation et comment avec l'expérience vécue du lien (Pinol-Douriez, 1984, p. 76-7)? 2) En quoi l'élaboration subjective des liens au génogramme est-elle significative de la création singulière que le sujet opère lorsqu'il met en tension ces deux ordres de savoir: il a une position déterminée culturellement (savoir conscient) en même temps qu'il a une place assignée et/ou désirée dans les liens intersubjectifs (savoir inconscient)?

Après un exposé succinct de la méthode d'analyse du génogramme que nous pratiquons, nous illustrerons par une situation clinique ces deux questions, manière de souligner en quoi notre motivation n'est pas de dire ce qu'il y aurait de pathologique dans telle ou telle mise en lien du sujet avec l'autre mais d'apprécier pourquoi l'on trouve cet aménagement subjectif plutôt qu'un autre, dans quelle mesure celui-ci est le fruit de l'élaboration de la part de dit et d'inédit sur le lien.

\section{Bref parcours notionnel}

En guise de prémisses nous proposerons de distinguer dans le lien de parenté ou familial deux aspects essentiels. Par le terme de lien nous faisons en effet référence:

1) D'une part à une relation entre deux individus, instituée par la parenté du sujet selon les règles de la culture. Le lien en ce sens de "mise en relation" situe le sujet par rapport aux autres et dit qu'elle est son identité symbolique (Aulagnier, 1975; Legendre, 1985, 1990). Il donne lieu le plus souvent à une connaissance d'ordre conscient.

2) D'autre part à une formation qui relie des sujets entre eux - au sens où le sujet est à la fois celui qui est assujetti à une place et qui se représente à partir de celle-ci. En ce sens le lien est une formation intermédiaire intersubjective (Kaës, 1994). Sous cet aspect second il relie des objets subjectivés et s'inscrit dans l'inconscient sous une forme mobilisable comme "représentation de l'être avec l'autre" à laquelle sont associées les valences affectives du lien: amour, haine, indifférence. L'inconscient s'inscrit, se re-présente et se dit dans le lien. L'expérience interactionnelle et affective avec l'autre de même que les identifications à l'autre et à partir de l'autre y travaillent et peuvent venir à se représenter notamment sous la forme du fantasme. En un mot le désir d'être avec l'autre et pour l'autre selon ce qu'en impose le lien intersubjectif et la dynamique intra-psychique font du lien une formation dynamique.

Nous venons d'esquisser la spécificité du lien: elle tient à son hétérogénéité même et à ce qu'elle a contribué à inscrire chez le sujet comme représentation 


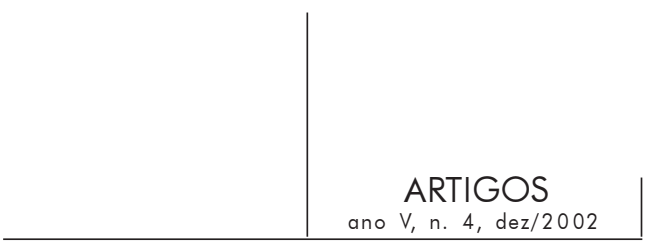

de l'autre et de lui-même. Cette formation intermédiaire entre le sujet et l'autre qui puise ses racines dans l'inconscient et qui tire sa raison d'être de l'institution culturelle ou de ce qui correspond à une institution dans le groupe familial est donc source et objet de conflit psychique. Nous le réduirons, pour des raisons de méthode au conflit ou à la tension entre d'une part ce que le lien édicte de la position du sujet et d'autre part ce qu'il assigne comme place identificatoire.

Le savoir conscient et inconscient que le sujet peut en élaborer dépend de cette hétérogénéité et de cette conflictualité. Pour le sujet le lien apparaît sous la forme d'un complexe représentatif, associant selon diverses modalités des aspects conscients, préconscients et inconscients. Il peut donc être appréhendé par le sujet lorsque celui-ci le représente au sens large sous deux modalités: une modalité consciente, secondarisée qui correspond à ce qu'il connaît de sa généalogie et des relations de parenté, une modalité représentative inconsciente fruit de la projection et/ou de la dénégation (Fédida, 1978) de sa place dans les liens. Ainsi le sujet se représente dans le lien et représente le lien sous ses aspects inconscients avec les modes de méconnaissance qui sont les siens. Néanmoins grâce à cette projection et à cette dénégation de sa place dans les liens le sujet peut élaborer psychiquement ce qui le situe dans son rapport inconscient avec l'autre.

Nous partons de l'idée que le conflit psychique s'exprime au génogramme par l'écart ou la disjonction entre d'une part la connaissance de sa position dans les liens et d'autre part la représentation de sa place dans le lien avec l'autre, représentation qui suppose quant à elle le travail de la pulsion, du fantasme, du désir. Ainsi dans ce cadre de définition et dans ce contexte clinique, le lien - du fait de ses caractéristiques propres - suscite une élaboration mentale comme psychique spécifique. Le conflit psychique à l'oeuvre donne à notre sens sa spécificité à l'élaboration subjective.

Nous définirons en conséquence l'élaboration subjective comme le travail propre au sujet qui transforme, transporte, articule ou disjoint les registres de savoir sur le lien. Elle désigne dans le contexte qui est le nôtre, les modes de traitement par le sujet de l'écart entre la connaissance de sa position dans la parenté (les déterminismes culturels et oedipiens) et la représentation de sa place dans les liens de désir aux autres. Elle serait caractéristique de la manière dont le sujet peut se situer à l'égard du savoir sur le lien: dans l'annulation des valences symboliques ou intersubjectives, dans la disjonction des registres de savoir ou dans l'articulation source de subjectivation des parts hétérogènes du lien.

L'élaboration subjective des liens au génogramme nous apparaît être un travail permanent d'ajustement entre la volonté consciente de se positionner dans les relations de parenté et de décrire son origine et sa filiation de ce point de vue et la motivation inconsciente de pervertir les places selon la place qu'occupe le 


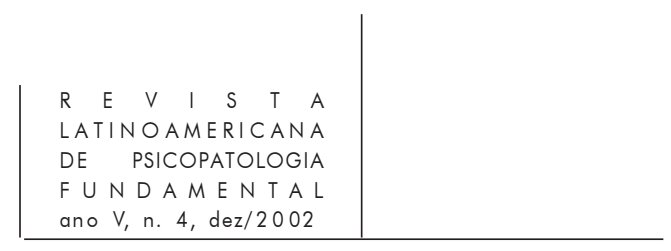

sujet dans le fantasme (fantasme originaire, roman familial, fantasme de filiation, fantasme d'identification). A ce titre nous conviendrons avec M.L. PradellesMonod (1999) que la position du sujet dans ses liens peut se dire de diverses manières et que le choix qu'opère le sujet parmi toutes les possibilités d'énonciation et de figuration dont il dispose est significatif du sens inconscient qu'il accorde à sa place dans les liens.

En résumé l'élaboration subjective des liens serait significative de la manière dont le sujet peut, en fonction de sa dynamique psychique, s'autoriser à penser, articuler, relier ce qui le détermine dans le lien avec l'autre.

\section{Abord méthodologique}

A partir des points que nous avons développé nous considérons que le génogramme constitue un "texte" signifiant où travaillent conjointement des processus secondaires et primaires; que l'implication subjective qu'il suscite met les sujets dans la situation d'avoir à élaborer des registres de savoir hétérogènes: des connaissances et des représentations inconscientes. En cela il peut être tenu pour une formation de compromis entre l'élaboration secondarisée de sa position dans les relations et l'élaboration inconsciente de sa place dans les liens.

\section{La connaissance de la position du sujet dans ses liens}

Nous abordons empiriquement la "connaissance" de la position du sujet dans ses liens à partir d'une analyse sémiotique de la forme "manifeste" de ce texte, qui se présente comme un mixte de signifiants iconiques et verbaux. L'élaboration sémiotique est appréciée à partir de deux pôles essentiels: le pôle de la différenciation et celui de l'identification. ${ }^{1}$ Sont ainsi relevés de façon systématique: le degré de différenciation des positions dans la parenté (du point de vue du code proposé, des sexes et des générations), le degré d'identification des liens enfin la qualité globale de l'élaboration des liens intra et intergénérationnels.

Ce premier temps de l'analyse de la forme sémiotique manifeste du texte génogramme, nous permet de déduire ce qu'il en est de la connaissance par le sujet de sa position dans la parenté du point de vue de la différenciation selon les sexes et les générations et de l'identification des relations de parenté (par les termes de parenté notamment).

1. Identification et différenciation sont ici entendus comme des processus logiques. 


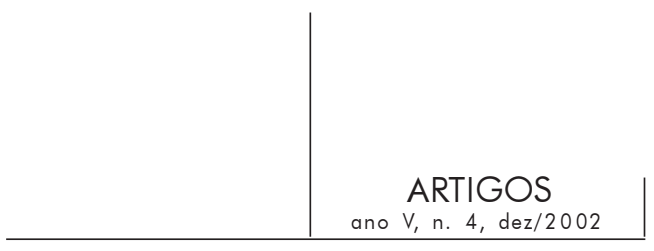

La représentation de sa place dans les liens

La production sémiotique, sous-tendue par des processus secondaires et primaires, est le matériau à partir duquel nous pourrons inférer la fantasmatique à l'œuvre dans les représentations de liens, apprécier en quoi celle-ci peut subvertir la connaissance dont le sujet dispose par ailleurs, saisir comment, dans certains cas, l'élaboration mentale de la double différence des sexes et des générations est fortement entravée. L'intérêt clinique de cette analyse sémiotique, et par conséquent des processus à l'œuvre dans l'élaboration subjective des liens, est bien de saisir ce qui fait "signe” dans ce texte singulier qu'est le génogramme, ce qui exprime la place inconsciente du sujet dans ses liens, ce qu'en un mot dit le sujet de sa place inconsciente dans les liens aux autres. Interroger la forme manifeste de ce "texte" vise à saisir le sens inconscient qui est à l'œuvre et les processus qui concourent à une telle sémiosis.

Concrètement la représentation de sa place dans les liens est appréciée à partir des signifiants (iconiques et verbaux) qui posent problème soit du point de vue de leur différenciation (exemple: confusion des sexes, des générations), soit du point de vue de leur identification (tout lien ou trait figuré dont la signification est ambiguë, voire confuse), soit du point de vue de leur formation (c'est le cas de tout signifiant dont les caractéristiques laissent à penser que le sujet démarque un sens précis par exemple en dissociant des lignées, en s'affiliant dans une uni-filiation, en sur-représentant un icône, etc.). Ainsi, toute production signifiante obéissant à une logique inconsciente qui mobilise le processus primaire (condensation, déplacement, surinvestissement etc.) est susceptible de susciter un travail d'interprétation du sens inconscient à l'œuvre.

Ces "signifiants" sont, à notre sens, spécifiques du travail du fantasme 2 autrement dit du travail des représentations inconscientes de sa place dans les liens. Nous les qualifions de signifiants analogiques de démarcation, à partir de la conception qu'en propose Rosolato $(1985,1992)$.

Parmi ces signifiants analogiques certains expriment à notre sens le désir du sujet, ce sont des signifiants "préférentiels". Il s'agit de ceux dont la facture ou la position ou la présence marquent une préférence de la part du sujet, peut s'y entendre une idéalisation du lien, un fantasme d'identification oedipien voire un fantasme de transmission, en un mot, les signifiants de démarcation du désir du sujet, qui, quoiqu'il en soit, parlent du lien avec l'autre sous son aspect

2. Soulignons (1) que par analogie avec le travail du rêve nous posons que le fantasme est présent aux deux extrémités du processus d'élaboration: d'une part il est lié au désir inconscient le plus profond, d'autre part il est présent dans l'élaboration secondaire; (2) que le fantasme est un point privilégié de saisi du passage entre le niveau psychique et le niveau mental. 


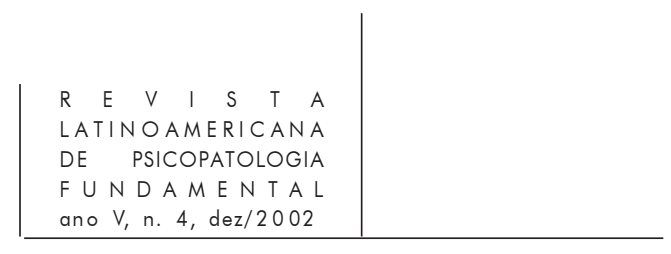

fantasmatique. Ces signifiants prennent en considération l'altérité quand bien même ils visent à dire un désir de ressemblance, de mêmeté, de filiation narcissique à l'égard d'un autrui de la parenté. Ils sont pris, à notre sens dans le conflit psychique ou la tension entre le même et l'autre. Ils témoignent du désir d'être dans le lien (exemple: le roman familial). Ils démarquent un sens inconscient pour le sujet, à cet égard nous les qualifions de signifiants de démarcation.

D'autres signifiants analogiques de démarcation nous semblent plus proches de la "représentation de chose", ce sont ceux dont la facture ou la position ou la présence marquent une énigme ou un impensable pour le sujet. Il s'agit par exemple de liens qui relient sans aucune distinction différentielle des membres mal connus par ailleurs. Ces signifiants nous semblent avoir une visée narcissique prévalente (cohésion, indifférenciation s'y entendent). Dans ce cas la démarcation, sous l'effet de la projection, ne prend pas en compte la différence des sexes et des générations, ce qu'ils expriment échappe au travail de la différenciation. La tension entre le même est l'autre y est absente, la représentation de sa place se dit au travers de fantasme d'auto-engendrement, de fantasmes utérins. La filiation biologique y prévaut sous la forme d'un impensable de l'engendrement par les deux sexes, la filiation narcissique quant à elle exclut tout écart entre générations voire l'inverse. En termes de roman familial nous avons affaire au roman familial du psychotique au sens d'A. Eiguer. Expression d'un même familial ou d'une étrangeté radicale ces signifiants de démarcation sont énigmatiques ${ }^{3}$ pour ne pouvoir être ni différenciés ni identifiés par le sujet. L'on s'attend à trouver de telles productions chez des sujets psychotiques mais aussi chez ceux pour lesquels la transmission de l'Oedipe est défaillante.

\section{Le rapport connaissance/représentation}

L'on s'attend à trouver une tension entre connaissance de sa position et représentation de sa place dans les liens variable, toutes choses égales par ailleurs, selon les sujets. Nous proposons de considérer que le rapport entre connaissance et représentation est significatif du rapport du sujet à l'Edipe. Dans le cadre de notre thèse nous cherchons à vérifier que la qualité de l'élaboration subjective tient aux types de fantasmes qui viennent donner sens aux événements connus par la famille (séparation, ruptures, recompositions etc.).

3. La définition des signifiants de démarcation suppose cette dimension de l'énigmatique, en ce sens toute expression analogique du désir du sujet est énigmatique, pour autant nous distinguons parmi les signifiants de démarcation au génogramme ceux dont le degré de différenciation témoigne d'un travail de l'Oedipe, de ceux qui évoquent la représentation de chose, l'originaire ou les proto-représentations. 


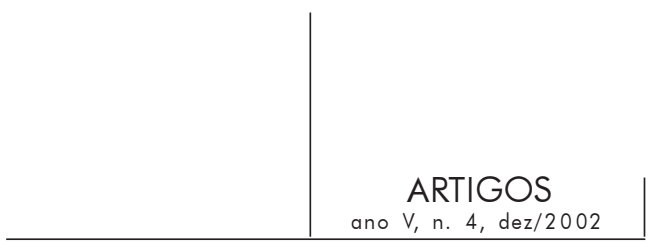

Illustration clinique de départ:

Nous présenterons un génogramme d'enfants afin d'illustrer notre question

L'élaboration subjective des liens serait-elle "menacée" par la nouveauté laissant le sujet dans un trop de liens inélaborables ou dans un défaut de repères symboliques?

Nous choisissons de parler de la figuration d'Elodie, 9 ans et demi, rencontrée dans le cadre scolaire et qui vit depuis l'âge de 5 ans avec son père suite à un divorce du couple parental; ce couple s'était formé après une première union de la mère qui a donné naissance à deux enfants; les difficultés éducatives de la mère dues à un alcoolisme chronique ont conduit le juge pour enfants à confier successivement chacun des enfants à leur père.

La réalisation du génogramme est très investie par cette enfant qui commence par figurer la lignée paternelle à partir de ses ascendants puis la lignée maternelle en suivant la même logique.

Très vite le "texte" se complexifie du fait de l'importance des membres figurés mais surtout de l'impossibilité pour le sujet de figurer ses parents dans leur triple position "d'enfant de", "de parents de" et d'alliés. Elle-même ne s'inscrit que dans la filiation paternelle. Le couple dissocié n'est absolument pas mis en rapport, toutefois les deux lignées sont contiguës.

A la fois riche et ambigu ce génogramme évoque un clivage des lignées de la filiation. Le rapport conflictuel entre connaissance de sa position et représentation de liens est notable au premier abord.

\section{L'élaboration sémiotique des liens}

\section{L'appartenance au groupe familial:}

Un écart manifeste existe entre la parole sur les liens et leur figuration analogique; constituée de familles, plus ou poins triangulées (avec enfants et parents), où seules les cellules familiales grand-parentales sont clairement figurées, le texte ne permet pas d'établir l'appartenance de chaque membre au groupe sur la seule base des liens intergénérationnels et intragénérationnels (alliance, collatéralité). La généralisation des liens est difficile.

Le degré de différenciation des sexes et des générations:

Dans ce texte riche le code est notablement enrichi par l'adjonction des noms de famille et des âges ainsi que par l'adjonction de symboles funéraires mais il est aussi transformé (des signifiants de démarcation, ex les cercles, de 


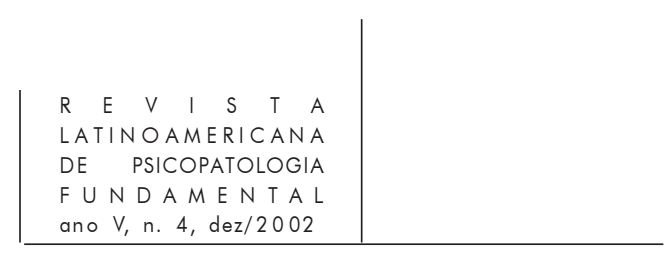

nombreux vides, blancs espaces, ainsi que des signifiants énigmatiques sont présents). Les symboles funéraires sont utilisés en premier lieu à propos de la sœur handicapée décédée du père puis seront attribués aux morts ou handicapés de la lignée maternelle.

\section{La différenciation des positions dans la filiation:}

La difficulté centrale pour Elodie est de différencier les positions selon les générations, non pas qu'elle ignore cette différence, en atteste la figuration des grands-parents et de leurs enfants, mais elle ne l'utilise pas ou mal dès lors qu'il s'agit de se positionner dans la filiation paternelle pour l'ensemble des membres figurés (12 “"pères' sont absents", des confusions dans les positions apparaissent pour ses cousins). L'ensemble est encore ambigu, l'on pourrait penser à partir de ce qu'elle en dit que la descendance est correctement pensée mais elle opère d'autres choix lorsqu'elle la sémiotise. Il est dans l'ensemble délicat de différencier les positions des enfants à l'égard de leurs parents, notamment de leur père.

Or c'est bien le problème psychique d'Elodie que de pouvoir différencier sa place dans sa propre filiation: elle n'est pas situable par rapport à sa mère en tant que mère, elle est située par rapport à son père mais dans une position relativement excentrée par rapport à ses grands-parents paternels, elle n'est pas situable par rapport à sa lignée maternelle.

\section{La différenciation des positions dans l'alliance:}

Les alliés sont rarement situés les uns par rapport aux autres. Le père et la mère ne sont pas repérables en tant qu'alliés dans cette famille où seule la sœur sur-représentée de la mère est inscrite dans une famille triangulée.

\section{La différenciation des positions dans la germanité:}

Même si Elodie figure des fratries complètes - hormis la sienne - les positions au sein des fratries sont parfois désordonnées, l'ordre de naissance n'est souvent pas respecté. De plus la figuration qu'elle adopte pour la fratrie de son père et de sa mère est source d'interrogations dans la mesure où nous $\mathrm{y}$ trouvons des signifiants de démarcation: des espaces entre parents et enfants pour le père, des enclaves pour la fratrie de la mère.

\section{Le degré d'identification des liens:}

Le degré d'identification sémiotique des liens est faible dans l'ensemble. Elodie énonce bien mieux les liens qu'elle ne les figure. Là encore l'on peut évoquer le travail des logiques inconscientes dans ses choix. Les liens figurés (dont certains sont "en trop") ne reposent qu'en partie sur les conventions culturelles proposées. Elodie invente plus qu'elle n' applique le code du lien. Ainsi 


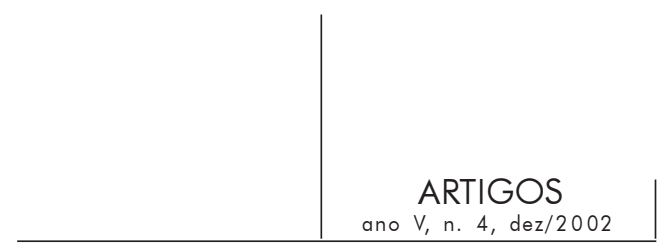

elle doit afin de figurer les liens dans la généalogie recourir à une duplication d'icônes (moyen d'isoler les positions de parent et d'enfant pour ses propres parents). Elle redouble également la soeur "centrale" de la mère. D'une lignée à l'autre, en dépit de différences dans la réalisation l'on constate que la filiation passe par certains membres mis en position préférentielle (son père, sa tante). Notons que de ce fait des liens supplémentaires sont présents entre frères et sœurs de père et de mère mais s'agit-il de liens de fraternité ou bien de l'expression d'un sens inconscient?

Domine dans ce texte une figuration du lien de filiation variable: parfois il attache deux sujets, parfois il est tendu vers un autre (le nom d'un autre) sans pouvoir s'attacher. La présence de ces deux modes distincts de figuration du lien ainsi que l'absence de certains d'entre eux nous donne à penser que l'identification du lien de filiation est variable selon les membres de sa généalogie.

En conclusion nous dirons que les représentations inconscientes semblent déterminer l'identification des liens de parenté dans les deux lignées même si Elodie connaît les termes de parenté et peut exprimer par ailleurs une assez bonne connaissance des règles de la parenté.

La connaissance de sa position dans les liens est au génogramme structurée par la double différence mais l'identification et la différenciation mentale de cette position est entravée par les logiques inconscientes. L'élaboration secondaire est freinée par une difficulté à penser la place des pères dans la généalogie et à combiner l'alliance avec la filiation. Sa connaissance de la lignée paternelle est plus affirmée que celle de la lignée maternelle.

La représentation de sa place dans les liens

La représentation de sa place pour Elodie:

Elodie se situe dans un lien exclusif au père, en bout de lignée, sur un plan décalé par rapport à ses germains. Cette filiation préférentielle d'allure oedipienne nous semble venir en défense face à la filiation maternelle. Ne dit-elle pas dans son génogramme sous la forme d'une dénégation: "ce n'est pas ma mère" combien il lui est difficile de penser ce lien et de se penser dans ce lien. La question que nous ouvrirons est donc de savoir si les fantasmes à l'œuvre ne remplissent pas une double fonction: d'une part maintenir, dans une logique oedipienne, le refoulement du lien de désir originaire à la mère, en déplaçant l'investissement libidinal sur le père, d'autre part d'éviter de se situer dans la filiation biologique du côté maternel, filiation où se transmettent mort et maladie. Elodie en effet distingue - et son angoisse lorsqu'elle l'évoque est patente - ce qui relève d'un accident: "la trisomie" de la sœur du père, de ce qui ressort de la maladie qui œuvre dans la lignée maternelle (cancer et handicap physique). 


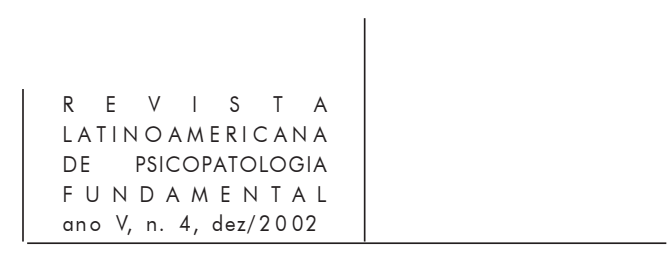

La représentation de la place du père dans ses liens:

Le père occupe un position préférentielle dans sa propre filiation, il est le seul au génogramme à s'inscrire dans une filiation par ses deux parents unis par un lien d'alliance. Les signifiants de démarcation présents à cet endroit (espace, liens au nom du père), ainsi que cette position centrale entre une sœur plus jeune et divorcée et une sœur aînée disparue, l'inscrivent dans un lien libidinal avec ces deux femmes. Par ailleurs il est figuré en position de père d'un couple de cousins et cousines, anormalement placés, dont Elodie méconnaît les noms et la filiation. Il apparaît dans une sorte de position de père utérin, à l'égard de sa fille ainsi qu'à l'égard de ces cousins dont l'énigmatique de la figuration (par rapport à l'ensemble de la production) autorise une interprétation.

S'agit-il pour Elodie de figurer une fratrie par le père, d'un désir de recomposition familiale ou s'agit-il plutôt de l'expression de fantasmes incestueux entre collatéraux?

Nous pencherions pour cette deuxième hypothèse explicative car la différenciation des générations est là confuse. Il nous semble qu'Elodie met ce père en position idéale de "père éducateur" du roman familial. Ce fantasme aurait donné sens à l'événement de sa séparation d'avec sa mère, et viendrait mettre en sens les liens du père aux autres comme celui qui soutient, étaye des femmesfilles fragilisées. Cette expression directe de l'Idéal du moi au travers du lien au père réel viendrait contrebalancer les fantasmes incestueux oedipiens.

La représentation de la place de la mère dans ses liens:

La mère en tant que telle est figurée à la fin du génogramme, isolée entre deux symboles funéraires. La figuration de cette mère parmi les morts ou ceux susceptibles de l'être puisqu'Elodie établit une analogie entre ces deux occurrences, signifie à la fois un vœu inconscient mais aussi une place dans les liens, une forme d'assignation identitaire.

La mère est secondairement inscrite dans sa filiation par le biais d'une sœur surinvestie (signifiant de démarcation). Au génogramme cette sœur vient en place de mère oedipienne (à la fois fille et enfant) et distincte de sa mère divorcée dont le désinvestissement par Elodie est manifeste. Toutefois le fantasme d'unifiliation par la mère est dominant même dans ce cas, plus nettement que dans la lignée paternelle. Le même dérive de la mère, des mères. La filiation biologique et narcissique ne passe pas par les pères.

Elodie ne peut articuler psychiquement les rapports de désir entre son père et sa mère. Son fantasme de scène primitive ne trouve à s'exprimer que par un déplacement sur la sœur de sa mère. Mais il se trouve que celui-ci vient rencontrer la question de l'origine biologique, de la transmission. A notre sens, la surreprésentation de cette cellule familiale, porteuse d'un mort, d'un enfant placé 


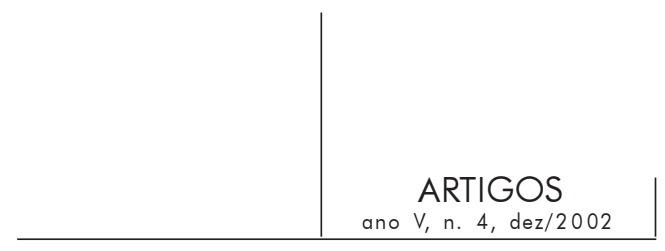

en institution et d'un enfant handicapé, la substitution qui la soutient expriment la rencontre conflictuelle entre les formes du lien de filiation (biologique, narcissique, instituée). Ne pas s'inscrire dans le lien à la mère, subjectiver la place de celle-ci comme "morte", et en rajouter dirai-je avec une figure de père éducateur, institué dans sa filiation, n'est-ce pas établir un compromis qui évite d'avoir à penser le lien à la mère dans sa complexité (institué en même temps que biologique et narcissique), n'est-ce pas éviter en se mettant en dehors de la lignée maternelle d'avoir à occuper à son tour une place de mort, de malade?

\section{Les représentations de liens au génogramme:}

En effet ce qui fait lien entre les deux lignées dont nous avons vu la particularité des représentations à l'œuvre ce n'est pas l'alliance mais ce qui, en termes de filiation biologique et psychique concerne un fantasme de transmission du même (source de rencontre fantasmatique de ce père et de cette mère?). Ce même est du registre de la maladie, de la mort du handicap. L'on comprend mieux la position excentrée, éloignée de tous de cette enfant. Toutefois nous avons vu que des aspects strictement oedipiens sont présents, en atteste la présence de signifiants de démarcation, les modes de défense, déplacement, dénégation, évitement du conflit oedipien en somme.

Les événements que sont la séparation, l'alcoolisme, la mort sont subjectivés par cette enfant au travers de fantasmes de mêmeté et de fantasmes oedipiens. Compte ténu de la facture générale du génogramme, du rapport connaissance/ représentation l'on peut considérer que l'élaboration subjective au génogramme est bien significative du rapport à l'Edipe de cette enfant. L'ambiguïté de la production tient à un évitement du lien identificatoire à la mère (oedipe inversé), lien qui s'il était subjectivé mettrait Elodie face à une place de "mort" dans sa généalogie. En termes d'économie psychique il s'agit à notre sens pour elle d'éviter le conflit oedipien et la réactivation des fantasmes originaires afin de vivre un lien éducatif "idéalisé” avec le père. En somme Elodie semble par là même éviter subjectivement les questions qui la travaillent: celle du sexe, de la castration (de la femme) et de la mort.

\section{Conclusion}

Quelle que soit la part de nouveauté des liens familiaux - nouveauté du côté de l'institution ou nouveauté du côté des interactions intersubjectives, il semble que pour la psyché il s'agisse d'une innovation. Face à l'inédit du lien la psyché aurait à faire ou bien avec de l'inconnu, de l'étrange, de l'inclassable ou 


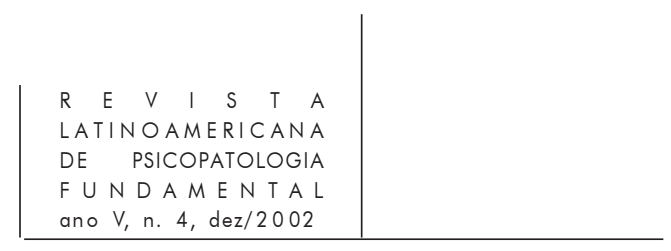

à l'inverse avec un trop connu (comme le serait un nouveau lien "narcissique" répétition d'un lien déjà connu).

Que le sujet parvienne à ramener l'inconnu à du connu en se fabriquant des frères ou de nouveaux papa et maman ou à l'inverse qu'il achoppe dans sa tentative de maîtrise de la part d'étrangeté du lien, se représentant par exemple dans un lien hors générations avec ce nouveau beau-père qui réveille néanmoins les pulsions en latence, il n'en demeure pas moins que la manière dont le sujet pense le lien - au sens freudien du terme - est significative de la création singulière qu'il a opérée et de la position subjective qu'il a adoptée face à l'inconnu, à l'inédit.

La situation clinique présentée vient souligner combien les événements familiaux font trace et prennent sens selon la dynamique psychique propre au sujet.

\section{References}

Aulagnier, P. (1975). La violence de l'interprétation. Paris: PUF, 1991.

Condamné à investir. In: Le trouble du penser. Paris: Gallimard. Nouvelle Revue de Psychanalyse, n. 25, p. 309-30, printemps 1982.

L'apprenti historien et le maître sorcier. Paris: PUF, 1984.

Assoun, P.L. Trouble du penser et pensée du trouble. Nouvelle Revue de Psychanalyse, n. 25, p. 77-106, printemps 1982.

L'entendement freudien. Paris: Gallimard, 1984, p. 295.

Psychanalyse. Paris: PUF, 1997.

Berthoud-Papandropoulou, I. et Thommen, E. Acquisition des relations de parenté chez l'enfant. Aspects logiques, lexicaux et énonciatifs. Bulletin de Psychologie, n. 60, p. 307-23, 1993.

Cannels, M. Un scénario, des acteurs et une mise en scène. Le génogramme et son processus. Revue Dialogue, n. 126, p. 53-63, $4^{\mathrm{èm}}$ trimestre, 1994.

CuYnEt, P. L'arbre généalogique en thérapie familiale psychanalytique. Le Divan Familial. p. 77-91, 1999.

Ducousso-Lacaze, A. et Grinom, M-J. Construction d'analogies en clinique psychanalytique, Psychologie de l'interaction, 2001 (sous presse)

Duret, I. et Lefebvre, A. Cherche vraie famille, à tout prix. Histoire d'une filiation honteuse, Cahiers de psychologie clinique, n. 9, p. 55-69, 1997.

FédIDA, P. L'absence. Paris: Gallimard, 1978.

Théorie des lieux dans la psychanalyse. In: Actualité des modèles freudiens, sous dir. P. Fédida et D. Widlöcher, Colloque de la Revue Internationale de Psychopathologie. Paris: PUF, p. 123-51, 1995.

Freud, S. (1900). L'interprétation des rêves. Trad. I. Meyerson. Paris: PUF, 1987. 


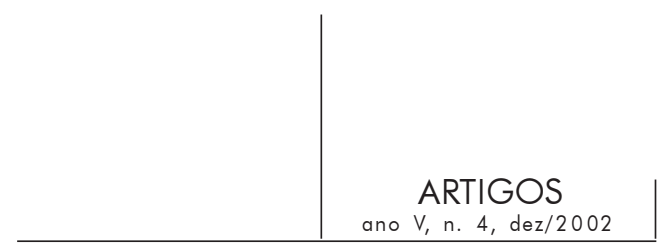

(1905). Trois essais sur la théorie de la sexualité. Trad. Ph. Koeppel. Paris: Gallimard, 1991.

(1908). Les théories sexuelles infantiles. Trad. J.Laplanche. In: La vie sexuelle. Paris: PUF, 1977, p. 14-27.

(1911). Formulation sur les deux principes de l'advenir psychique. Euvres complètes. Trad. coll. Paris: PUF, 1998, p. 11-21. v. XI.

(1912-1913). Totem et tabou. Trad. Jankélévitch. Saint Amand: Payot, 1980.

(1914-1915). CEuvres complètes. Trad. coll. Paris: PUF, 1988. v. XIII.

(1921- 1923). Euvres complètes. Trad. coll. Paris: PUF, 1991. v. XVI.

(1939). L’homme Moïse et la religion monothéiste. Trad. C. Heim, Paris:

Gallimard, 1989.

Guyotat J. Mort, naissance et filiation. Paris: Masson, 1980.

Filiation et puerpéralité. Logiques du lien. Paris: Masson, 1993.

Sujet de la filiation, filiation du sujet. Revue Internationale de Psychopathologie, n. 13, p. 5-14, 1994.

Halimi, Y. et Kномsi, A. Savoirs et structures architecturales de parenté. L'information psychiatrique, n. 2, février/1991.

Hurstel, F. La famille après le divorce. Dialogue, $4^{\text {ème }}$ trim./1994.

KAËs, R. Les organisateurs psychiques du groupe. GRUPPO, n. 2, p. 117-25, 1986. Les organisateurs psychiques du groupe. GRUPPO, n. 3, p. 113-23, 1987.

Pacte dénégatif et alliances inconscientes. GRUPPO, n. 8, Secrets de famille et pensée perverse, Apsygée, 1992.

Le groupe et le sujet du groupe. Éléments pour une théorie psychanalytique du groupe. Paris: Dunod, 1993a.

Transmission de la vie psychique entre générations, art. Introduction au concept de transmission psychique, p. 17-32, art. Le sujet de l'héritage p. 1-15, Paris: Dunod, 1993b.

La parole et le lien. Paris: Dunod, 1994.

LACAN, J. (1956-57). Le séminaire. Livre IV. La relation d'objet. Paris: Seuil, 1994.

(1958). Le séminaire. Livre V. Les formations de l'inconscient, leçon du 15.1.1958, Champ Freudien, Paris: Seuil.

Laflaquiere, A. Les destins de l'analogie. Bulletin de psychologie, 2001 (sous presse).

Laplanche, J. et Pontalis, J.-B. Fantasme originaire, fantasme des origines, origines du fantasme. Paris: Hachette, 1985.

Legendre, P. Leçons IV l'inestimable objet de la transmission. Paris: Fayard, 1985. Leçons IV, suite 2. Paris: Fayard, 1990.

Lemaire-Arnaud, E. Utilité du génogramme pour la mise au jour des phénomènes transgénérationnels, Dialogue, recherches cliniques et sociologiques sur le couple et la famille, p. 3-7, $3^{\text {ème }}$ trim./1980.

"Le génogramme et les secrets de famille". Dialogue, n. 80, 1985.

LYNCH, E. \& B. Utilisation du génogramme comme outil de thérapie et de diagnostic 


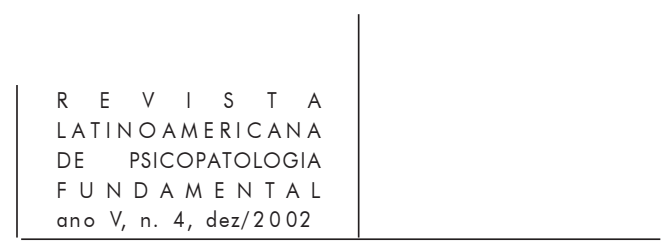

familial, extrait de Family assessment, Document interne de "Institute for human development, Bradford, Connecticut. Trad. J.M. Robine.

Mc. Goldrick M. et al. Génogrammes et entretien familial. Paris: E.S.F., 1980.

Papageorgiou-Legendre. Fondement généalogique de la psychanalyse. Paris: Fayard, 1990.

Pinol-Douriez, M. Bébé agi-Bébé actif. Paris: PUF, 1984, p. 233.

Pulsions, représentations, langage. Textes de base en psychanalyse, DelachauxNiestlé, Paris, 1997.

Pradelles-Monod M.-L. La grand-mère, la mère et la fille. Quand des femmes s'approprient leur héritage familial ou la généalogie "imaginarisée". Dialogue, recherches cliniques et sociologiques sur le couple et la famille, p. 64-90. $4^{\text {ème }}$ trim./1994.

Transmissions généalogiques: de l'événement à la trace de l'événement. Revue Internationale de Psychopathologie, n. 24, p. 33-46, 1997.

Le récit d'une migration: les traces d'un déplacement. Cliniques Méditerranéennes, n. 55/56, 1998.

La construction du lien de filiation entre trois générations de femmes. Repères pour une analyse clinique d'entretiens de recherche. Thèse pour le Doctorat d'Etat, 1999.

Sous la filiation: 1'ancestralisation. In: Filiations 1, Cliniques méditerranéennes, n. 63, 2001.

Les "constructions filiatives": une formation de compromis? Evolution Psychiatrique, n. 66, p. 105-10, 2001.

Rosolato, G. Éléments de l'interprétation. Paris: Gallimard, 1985.

La filiation: ses implications psychanalytiques et ses ruptures. Revue topique, n. 44, p. 187-200, 1989.

Comment s'isolent les signifiants de démarcation. Revue Topique, n. 49, p. 6579, 1992.

Les fantasmes originaires et leurs mythes correspondants. Nouvelle Revue de Psychanalyse, n. 46, 1992.

SAntelices, M-P., Simon, M., Chouvier, B. Utilisation libre du génogramme en pratique clinique auprès d'enfants placés. Le divan familial, 1999.

\section{Resumos}

O autor propõe abordar a eventual "novidade" de laços familiares para o psiquismo, a partir do que as crianças-sujeito podem elaborar subjetivamente. Desenvolve-se aí a idéia segundo a qual a qualidade da elaboração subjetiva dos laços de parentesco ao genograma é significativa da maneira pela qual o sujeito subjetiva 


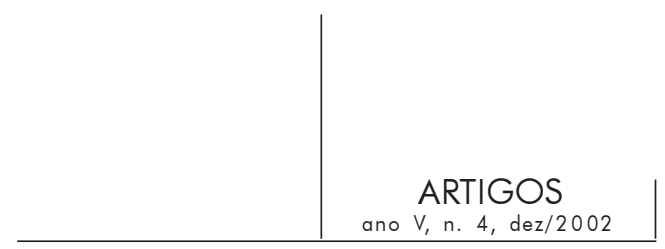

a sua posição instituída e seu lugar inconsciente no laço com o outro. É evidenciado, a partir de uma situação clínica, que os modos de tensão entre conhecimento $e$ representação dos laços são significativos do trabalho de Édipo para o sujeito.

Palavras-chave: Elaboração subjetiva, laço, conhecimento, representação, genograma

El autor propone abordar la eventual "novedad" de las ligaciones familiares para el psiquismo a partir de lo que los niños-sujetos pueden elaborar subjetivamente de ellas. Desarrolla la idea, según la cual, la cualidad de la elaboración subjetiva de las ligaciones de parentesco al genograma es significativa de la manera por la cual el sujeto subjetiva su posición instituida y su lugar inconciente en el lazo con el otro. Resalta a través de una situación clínica que los modos de tensión entre conocimiento y representación de las ligaciones son significativos del trabajo del Edipo en el sujeto.

Palabras claves: Elaboración subjetiva, ligación, conocimiento, representación, genograma

This article discusses possible "novelties" in family ties for the psychism, based on what children-subjects can work through subjectively. The idea developed here is that the quality of the subjective working through of kinship ties in reference to the genogram is closely related to the way in which the subject subjectivates her instituted position and her unconscious place in the ties with the other. Based on a clinical situation, it is shown that the modes of tension between knowledge and representation of the ties are closely related to the subject's Oedipal development.

Key words: Subjective working through, tie, knowledge, representation, genogram

Versão inicial recebida em junho de 2001

Aprovado para publicação em novembro de 2002 


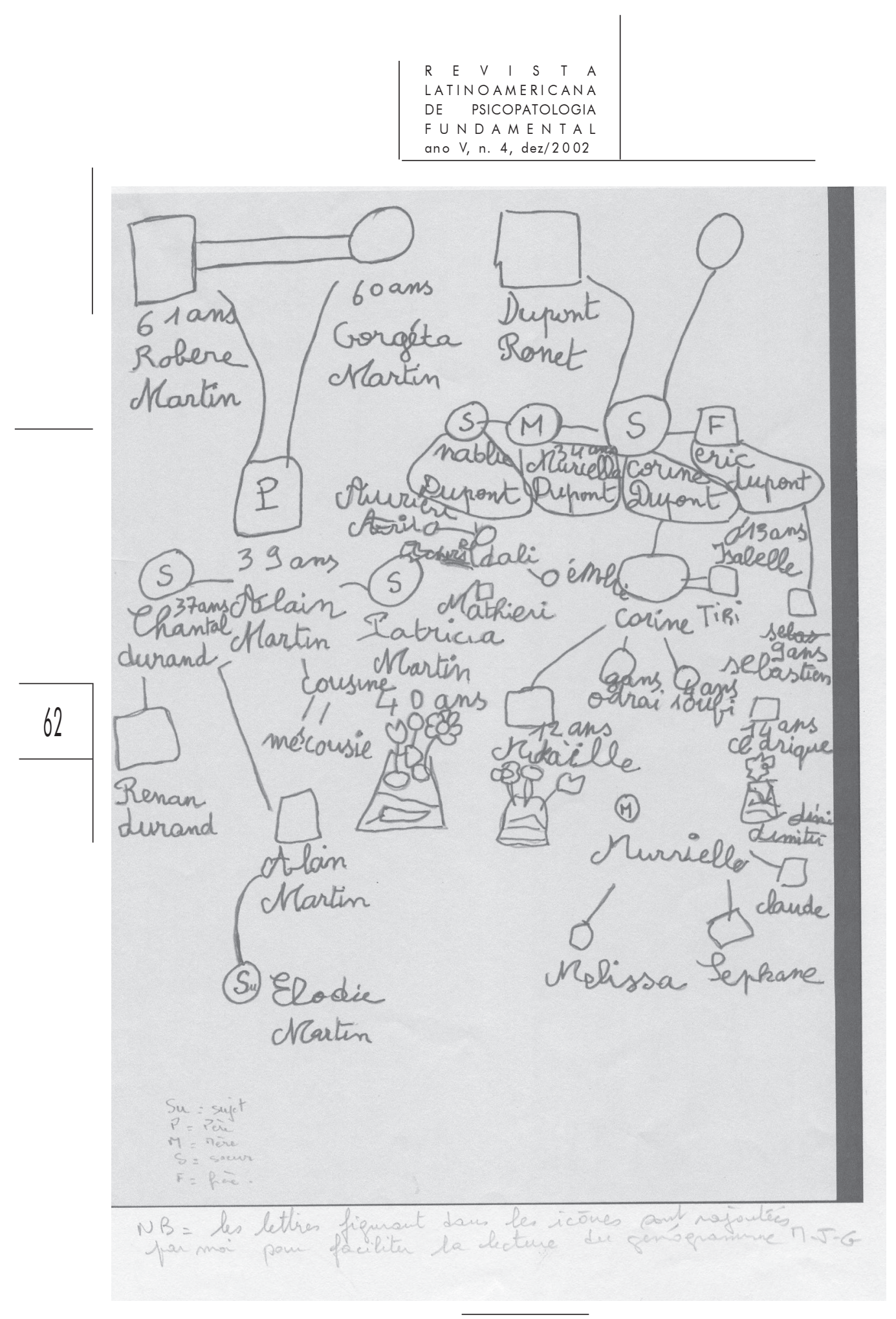

